Ann. Génét. Sél. anim., I97I, 3 (4), 497-507.

\title{
MÉTHODES D'ÉTABLISSEMENT DES INDEX DE SÉLECTION DES TAUREAUX DE RACES A VIANDE SUR LA VALEUR ÉCONOMIQUE DE LEURS DESCENDANTS
}

\author{
J.-L. FOULLEY et R. ROUVIER \\ Station de Génétique quantitative et appliquée, \\ Centre national de Recherches zootechniques, I. N.R. A., \\ 78 - Jouy-en-Josas \\ Laboratoire de Méthodologie génétique, \\ Centre de Recherches de Toulouse, I. N. R. A., \\ B. P. 12,31-Castanet
}

\section{RÉSUMÉ}

La sélection des taureaux de races à viande destinés au croisement de première génération a visé jusqu'ici à maximiser la recette brute fournie par les veaux croisés à un âge donné. Diverses méthodes d'indexation utilisant l'information fournie par les contrôles d'aptitude ont été discutées ainsi que l'incidence de l'adjonction d'une contrainte de progrès génétique nul sur le poids à la naissance des veaux. Une application numérique faite dans les races Charolaise et Limousine a permis de mettre en évidence des différences de comportement entre les deux races sous l'effet d'une sélection par index ; ainsi, le coût génétique de la contrainte est apparu plus élevé en Limousin qu'en Charolais.

\section{I. - POSITION DU PROBLÈME}

Le développement du croisement commercial entre taureaux de races à viande et femelles de races laitières ou rustiques en vue de la production de veaux de boucherie a entraîné le développement de schémas de sélection des mâles utilisés par les centres d'insémination sur les caractères de croissance et de conformation bouchère. Cette sélection intéresse successivement les performances de l'individu puis celles de sa descendance croisée (FREBLING et GAILIARD, I970). Dans le dernier cas, on estime, pour chaque taureau, son aptitude générale au croisement après une correction des données destinée à éliminer les effets liés à une répartition déséquilibrée 
des sexes et races maternelles dans chaque lignée paternelle (VISSAC, I964). Les trois critères considérés dans cette estimation sont :

- le poids vif à 75 jours (âge auquel sont abattus les premiers veaux), déterminé par intrapolation linéaire entre deux pesées ;

- le pointage de valeur bouchère égal à la somme de cinq appréciations subjectives de la musculature, de la finesse du squelette, du degré de rétraction du ventre, de l'état d'engraissement et de l'intensité de décoloration des muqueuses supposée liée à celle du muscle ; ces notes sont données lors de la dernière pesée de l'animal à un âge de deux mois et demi à trois mois ;

- le prix de vente au kilogramme vif fourni par l'éleveur; dans la mesure où, comme c'est en général le cas, les veaux relatifs à une série de taureaux contemporains sont nés et commercialisés dans une période de temps et une région limitées, ce prix qui constitue l'élément de la transaction, peut être considéré, en effet, comme représentatif de la conformation bouchère du veau au moment de la vente.

L'objectif global de sélection doit tendre à améliorer la rentabilité de la spéculation animale entreprise par l'éleveur. Dans le cas de la production de veaux de boucherie effectuée dans les régions du centre et du sud-ouest de la France, il est difficile d'appréhender cette rentabilité, étant donné les variations considérables des techniques de production : allaitement maternel au pis ou au seau, avec ou sans adjonction d'une mère nourrice ou d'aliment d'allaitement. Les âges et poids d'abattage varient de deux à cinq mois et de $\mathrm{I} 20$ à $250 \mathrm{~kg}$ respectivement, en fonction de la demande du marché local, de contingences liées à l'éleveur et indépendantes de l'animal, du potentiel de croissance et de l'état d'engraissement de ces derniers. On peut en fait considérer que, dans la majorité des cas, le veau consomme le lait maternel, les quantités non consommées n'étant pas valorisées. La recette brute qui s'exprime par le produit du poids de vente par le prix de vente est alors le meilleu rcritère de rentabilité de la production de veaux de ce type. Si l'on admet en outre que les différences moyennes d'âge de vente entre descendances paternelles sont négligeables on peut retenir comme critère global de sélection le produit des valeurs génotypiques relatives au poids à 75 jours et au prix de vente.

Si ce critère a été utilisé dans un premier temps, le calcul des paramètres génétiques (coefficients d'héritabilité et de corrélation génétique) entre les trois variables considérées et le poids à la naissance (POUJARDIEU et VISSAC, I968) conduit à effectuer certaines critiques quant à son efficacité.

a) L'augmentation du poids à 75 jours risque d'entraîner indirectement une augmentation du poids à la naissance dont l'héritabilité est en général plus élevée que celle du poids à 75 jours - en race Charolaise notamment - et de la fréquence des difficultés de mise bas des veaux croisés ; ces difficultés sont, en effet, étroitement liées, entre descendances paternelles, au poids des veaux à la naissance (BELIC et MENISSIER, Ig68).

b) La substitution de la note de pointage au prix de vente dont 1'héritabilité est plus faible permettrait d'améliorer la précision d'estimation de la conformation bouchère et l'efficacité de la sélection.

La prise en compte de ces remarques et, d'une façon plus générale, la définition d'un objectif global de sélection adapté à la situation qui nous préoccupe, soulèvent le problème de l'introduction, d'une part de caractères à seuil - cas du poids à la 
naissance des veaux dont l'incidence sur les difficultés de mise bas pour une femelle donnée se manifeste suivant un ou plusieurs seuils - d'autre part de fonctions quadratiques des caractères dans les index de sélection. Si l'emploi de contraintes (CuNNINGHAM, MOEN et GJEDREM, I970) dans l'établissement de ces index (limitation du progrès sur le poids à la naissance à une valeur fixée par exemple) fournit une solution adaptée dans un premier temps pour le premier problème, la sélection en faveur du produit de deux caractères n'a pas encore reçu de solution pratique satisfaisante.

Après avoir discuté les méthodes générales d'élaboration des index de sélection dans le cas d'un objectif quadratique, nous appliquerons à la sélection sur descendance croisée des taureaux de races à viande divers types d'indexation tenant compte partiellement ou totalement des critiques formulées ci-dessus.

\section{II. - DISCUSSION DU CHOIX D'UN INDEX DE SÉLECTION}

\section{CHEZ LES BOVINS, DANS LE CAS D'UN OBJECTIF QUADRATIQUE}

Les index de sélection sur plusieurs caractères onc surtout été étudiés dans le cas simple où l'index est un prédicteur d'une combinaison linéaire $\mathrm{H}$ des valeurs génétiques additives à améliorer (SMIтH, I936 ; HAzEL, I943; RouviER, I969a).

En production de viande bovine, les comparaisons ont concerné généralement des index établis sur cette hypothèse de linéarité ; (LEHMAN et al., I96I) pour la production de veaux de races anglo-saxonnes; (LINDHOM et STONAKER, I957; WIL,SON et al., 1963) pour des bovins Hereford plus âgés. En réalité, il est de nombreux cas où cette condition n'est pas vérifiée, ainsi que l'ont souligné KEMPTHORNE (1957), MoAV (I966), Wilton et VAN VILECK (I967, I968, I969), Harris (I970).

Avec un critère purement multiplicatif, il est possible de se ramener à une fonction linéaire en prenant les logarithmes (EILSTon, I963; Smrтh, I967). Cela nécessite une analyse des variances et covariances des logarithmes des valeurs phénotypiques qui n'a d'intérêt que si leur distribution peut être considérée comme normale. Or rien ne nous permet, notamment dans notre cas, d'accréditer plus cette hypothèse de log-normalité que celle de normalité (Rouvier, I $969 b$ ).

Récemment, Wilton, Evans et VAN Vleck (I969) ont abordé cette question de façon générale et WiLTON, VAN VLECK (I968, I969) en ont donné des exemples pour la sélection des vaches et des taureaux laitiers. Ces auteurs ont établi, par la méthode de minimisation de la variance résiduelle, deux index : l'un linéaire, l'autre quadratique. $\mathrm{L}_{\mathrm{a}}$ valeur génotypique globale $\mathrm{H}$ est de la forme :

$$
\mathrm{H}={ }^{t} a(\mu+g)+{ }^{t}(\mu+g) \mathrm{A}(\mu+g)
$$

où

$\mu+g$ désigne le secteur $(m, I)$ des valeurs génétiques additives des $m$ caractères considérés dans la sélection, $\mu+g$ ayant une distribution $\mathrm{N}(\mu, \Sigma g)$,

$a$ le vecteur $(m, \mathrm{I})$ des pondérations de ces valeurs génétiques;

A la matrice carrée $(m, m)$ symétrique des pondérations des carrés et des produits deux à deux des $m$ valeurs génétiques additives. 
La meilleure prédiction par un index linéaire s'écrit :

où

$$
\mathrm{I}_{l}=\alpha_{l}+{ }^{t}(a+2 \mathrm{~A} \mu) \hat{g}
$$

$\alpha_{l}$ est une constante

$\hat{g}$ la variable aléatoire $\mathrm{E}(g / p)$ espérance conditionnelle de $g$ quand $p$ varie. $p$ vecteur $(n, \mathrm{I})$ des observations phénotypiques exprimées en termes de déviation par rapport aux moyennes $\mu$, la distribution de $p$ étant supposée $\mathrm{N}\left(\mathrm{o}, \Sigma_{p}\right)$.

On démontre que $\mathrm{E}(g / p)=\sum_{g p} \sum_{p}^{-1} \cdot p$ où $\sum_{g p}=\mathrm{E}\left(g^{t} p\right)$ matrice $(m, n)$ de terme général $\operatorname{cov}\left(g_{i}, p_{j}\right)$

La meilleure prédiction par un index quadratique est:

$$
\mathrm{I}_{q}=\alpha_{q}+{ }^{t}(a+2 \mathrm{~A} \mu) \hat{g}+{ }^{t} \hat{g} \mathrm{~A} \hat{g}
$$

où $\alpha_{q}$ est une constante et $\hat{g}$ à la même signification que précédemment.

Cette expression équivaut, à une constante près, à l'estimation du maximum de vraisemblance de $\mathrm{H}$ connaissant $p$ notée $\mathrm{H} / p$ et à l'espérance conditionnelle de $\mathrm{H}$ connaissant $p$.

L'index linéaire équivaut aux recommandations de SMITH (I967), le quadratique à celles de HARRIS (I970) qui préconise, plus généralement, d'utiliser comme critère sélectif dans le cas d'une fonction complexe d'objectif, $H=\left(G_{1}, G_{2},-G_{n}\right)$, l'index $I=H\left(I_{1}, I_{2},-I_{n}\right)$ obtenu en remplaçant les $G_{i}$ par leurs prédicteurs $I_{i}$ sur les valeurs phénotypiques observables.

Dans la discussion de ces méthodes de prédiction, il importe de préciser les deux points suivants :

a) pour que 1'index $\mathrm{I}_{q}$ soit applicable, il faut que l'espérance du progrès génétique s'exprime de façon simple à partir de $\mathrm{I}_{q}$. Nous avons de fait vérifié que cette espérance sur l'objectif global de sélection quadratique $\mathrm{H}$ était bien égale, dans une population idéale de très grand effectif où tous les types d'accouplement sont équiprobables et pour des caractères polygéniques ne présentant pas d'épistasie, à la différence entre les valeurs moyennes de l'index dans la population des parents après et avant sélection ;

b) la variable $I_{q}$, explicitée en (3), a une loi qui ne peut être normale si $p$, vecteur des variables phénotypiques prédictrices centrées 1'est. Le calcul de son espérance sur la population tronquée des animaux sélectionnés ne peut alors donner lieu à une expression simple en fonction de l'écart-type, $\sigma \mathrm{I}_{q}$ et de l'intensité $i$, fonction du pourcentage d'animaux sélectionnés. La validité d'une sélection sur ce type d'index est donc discutable. On ne peut admettre que l'espérance du progrès génétique sur $\mathrm{H}$ est $i \sigma \mathrm{I}_{q}$ ainsi que le font WILTON et VAN VLECK (I968, Ig69) si l'importance de 1'erreur que fait commettre cette approximation n'est pas appréciée. Il se peut qu'elle soit faible - mais rien ne nous permet de l'affirmer a priori - et même négligeable par rapport aux autres erreurs faites, notamment celles inhérentes à l'emploi de matrices $\Sigma_{g}, \Sigma_{p}, \Sigma_{g p}$ estimées et non connues a priori (WILLIAMs, I962). La simulation devrait être un moyen d'appréhender ce problème. 


\title{
III. - COMPARAISON DE DIVERS SYSTÈMES D'INDEXATION DES TAUREAUX
}

\author{
SUR LA VALEUR DE LEURS DESCENDANTS
}

\section{a. - Méthodes d'étude et comparaisons réalisées}

Dans 1'état actuel, plusieurs attitudes sont possibles :

- sélectionner directement sur la valeur du produit exprimant la recette brute mais il est facile de vérifier qu'une telle sélection indirecte, que ce soit pour le produit " poids à 75 jours $\times$ prix unitaire de vente " ou " poids à 75 jours $\times$ note de pointage " est moins efficace qu'une sélection sur index combinant les deux caractères impliqués dans le produit, voire même qu'une sélection sur un seul caractère, celui de plus forte héritabilité ;

- aborder le problème à la façon de KEMPTHORNE et NoRDSKOG (I959) en considérant les termes produits au carré tels que $g_{i} \times g_{j}, g_{i}$ comme de nouvelles caractéristiques génétiques dont on calcule les variances et covariances et se ramener ainsi à l'établissement d'un index linéaire ;

- ou, vu la complexité des lois des variables quadratiques, prendre 1'approximation linéaire $\mathrm{H}_{l}$ comme le fait $\mathrm{SmIsm}_{\text {( }}$ g67) dont l'index $\mathrm{I}_{l}$ défini par WILton et VAN VLECK est le meilleur prédicteur.

Le troisième procédé paraît le plus judicieux et c'est donc sur cette base qu'ont été construits, dans notre étude, les divers systèmes d'indexation des taureaux soumis au contrôle de descendance. Compte tenu des deux aspects qui nous préoccupent dans l'indexation de ces taureaux utilisés en croisement et qui concernent, outre le type de fonction utilisée, le choix du critère d'appréciation de la valeur bouchère et l'incidence de l'effet direct du taureau sur la fréquence des difficultés de vêlage, nous avons comparé les index suivants :

I. les index $I_{1}$ prédicteurs centrés de l'approximation linéaire $\mu_{2} \mu_{3}+\mu_{3} g_{2}+\mu_{2} g_{3}$ du produit $\left(\mu_{2}+g_{2}\right)\left(\mu_{3}+g_{3}\right)$ des valeurs génétiques additives non centrées du poids à 75 jours $\left(\mu_{2}+g_{2}\right)$ et du pointage $\left(\mu_{3}+g_{3}\right)$, comme 1'a suggéré VISSAC (I964); ce produit présentant l'avantage de ne pas être lié aux fluctuations conjoncturelles de prix.

2. les index $I_{2}$ prédicteurs centrés de 1'approximation linéaire $\mu_{1} \mu_{2}+\mu_{1} g_{2}$ $+\mu_{2} k g_{3}$ du produit $\left(\mu_{2}+g_{2}\right)\left(\mu_{1}+k g_{3}\right)$ de la valeur génétique additive du poids à 75 jours par la régression $\mu_{1}+k g_{3}$ de la valeur génétique additive au prix unitaire de vente $\mu_{1}+g_{1}$ sur le pointage centré $g_{3}$.

Dans chacun des deux cas, nous avons étudié, en outre, 1'influence de l'adjonction de la contrainte : " maintien du poids à la naissance constant" $\left(\mathrm{E}\left(\Delta_{g_{4}}\right)=\mathrm{o}\right)$ en utilisant la méthode de calcul des index avec contrainte, proposée par CunNINGHAM, MOEN et GJEDREM (I970). Les calculs ont été effectués à partir des matrices de variances et covariances génétiques $\Sigma_{g}$ et phénotypiques $\Sigma_{\bar{p}}$, déduites de l'étude biométrique de Poujardieu et Vissac (I968). 


\begin{tabular}{|c|c|c|c|c|c|c|c|c|c|}
\hline & 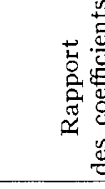 & & 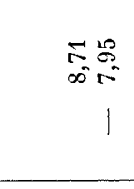 & & 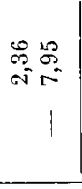 & 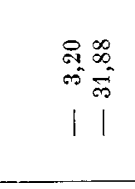 & & 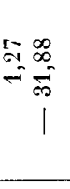 & \\
\hline & 造 & & $\begin{array}{l}\therefore: 0 \\
\therefore: \\
0\end{array}$ & & $\begin{array}{l}\infty \\
0 \\
0 \\
0 \\
0 \\
0\end{array}$ & 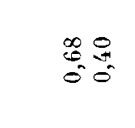 & & $\begin{array}{ll}\infty & 0 \\
0 & \stackrel{N}{0} \\
0 & 0\end{array}$ & \\
\hline & 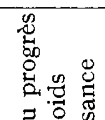 & $\begin{array}{l}a^{\circ} \\
\bar{y}\end{array}$ & $8^{\circ}$ & & $8^{\circ}$ & $\stackrel{8}{\circ}^{\circ}$ & & $\S^{\circ}$ & \\
\hline $\begin{array}{l}\overrightarrow{0} \\
0 \\
: \\
: 1 \\
0 \\
0 \\
0 \\
0\end{array}$ & 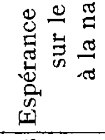 & 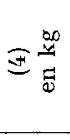 & $\begin{array}{c}200 \\
0 \\
0\end{array}$ & & $\begin{array}{l}8 \\
\therefore 0 \\
0\end{array}$ & 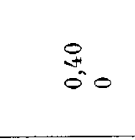 & & $\begin{array}{l}\hat{1} \\
\text { ing } \\
\text { की }\end{array}$ & \\
\hline 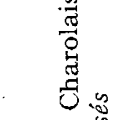 & 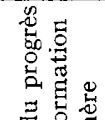 & $\begin{array}{l}\circ \\
8 \\
8\end{array}$ & $\stackrel{g}{g}$ & & $\underset{6}{8} \stackrel{10}{*}$ & छ욤 & & $\stackrel{8}{8}$ & 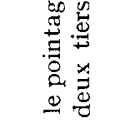 \\
\hline 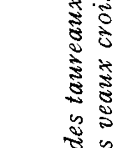 & 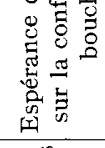 & 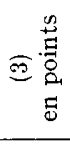 & 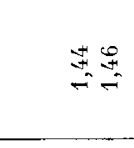 & & $\begin{array}{l}\mathrm{A} \\
\mathrm{N} \\
=\end{array}$ & 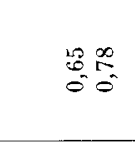 & & 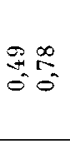 & 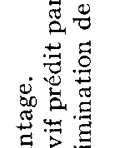 \\
\hline 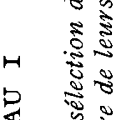 & 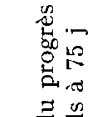 & $\begin{array}{l}\circ \\
5\end{array}$ & $\oint_{3}$ is & & $\stackrel{\sim}{\Im}$ & $8 \stackrel{8}{\circ}$ & & $\stackrel{8}{g}$ & 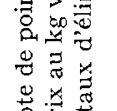 \\
\hline 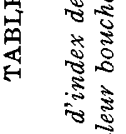 & 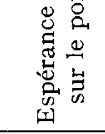 & $\widehat{\sigma}_{\vec{d}}^{\infty}$ & $\begin{array}{l}\approx: \\
=0 \\
=0\end{array}$ & & $\begin{array}{l}\stackrel{9}{:}: 0 \\
\stackrel{8}{*}: 0\end{array}$ & $\begin{array}{l}\overline{5} \quad \bar{b} \\
\text { is }\end{array}$ & & 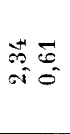 & 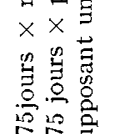 \\
\hline 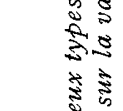 & 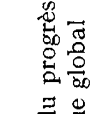 & $\begin{array}{l}\partial^{\circ} \sigma \\
\tilde{\partial}\end{array}$ & $8 \infty$ & & $\stackrel{8}{g}$ & 8 in & & $\underset{g}{8}$ it & 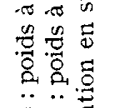 \\
\hline 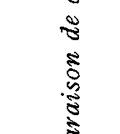 & 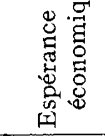 & 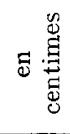 & 눙용 & & 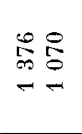 & 总哭 & & 㝵量 & 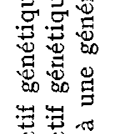 \\
\hline है & 蒫 & & 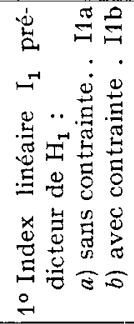 & 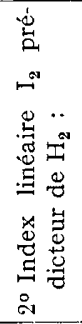 & 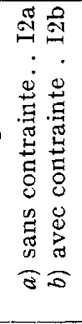 & 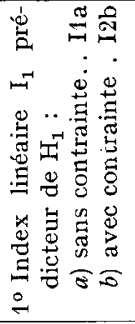 & 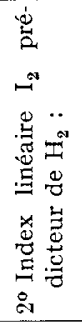 & 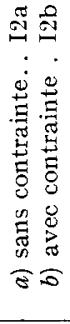 & 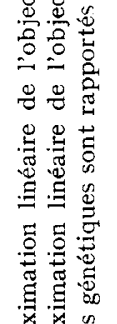 \\
\hline & 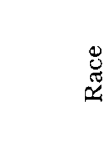 & & & 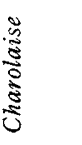 & & & 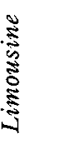 & & 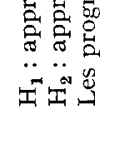 \\
\hline
\end{tabular}




\section{b. - Résultats et discussion.}

Nous avons rassemblé sur le tableau I les critères suivants qui expriment l'orientation et l'efficacité de la sélection correspondant à chaque type d'index :

- les gains génétiques globaux et sur chaque caractère avec une intensité de sélection égale à l'unité;

- le rapport $b_{3} / b_{2}$ des coefficients du pointage et du poids à 75 jours dans 1 'index ;

- le carré $\mathrm{R}^{2}(\mathrm{H}, \mathrm{I})$ du coefficient de corrélation entre 1 'index et la valeur génotypique globale correspondante qui mesure la précision de l'index.

La comparaison intra-race de pères, des index $\mathrm{I}_{1} a$ et $\mathrm{I}_{2} a$ non contraints, ne laisse pas apparaître de différences marquées. L'index $I_{1}$ est légèrement pénalisé quand on considère le progrès génétique global ( $\mathrm{I}_{3} \mathrm{I}_{4}$ pour $\mathrm{I}_{1} a$ contre $\mathrm{I}_{37} 6$ pour $\mathrm{I}_{2} a$, en Charolais, I 339 contre I 400 en Limousin). L'index $\mathrm{I}_{1} a$ attribue un peu plus d'importance à la note de valeur bouchère que ne le fait $\mathrm{I}_{2} a$, comme le montrent les valeurs prises par le rapport $b_{3} / b_{2}(8,7 \mathrm{I}$ contre 2,$36 ; 3,20$ contre $\mathrm{I}, 27$ respectivement en Charolais et Limousin) et les progrès respectifs sur les caractères de pointage et de croissance : $\left(\mathrm{I}, 44\right.$ points et $\mathrm{I}, \mathrm{I} 7 \mathrm{~kg}$ à 75 jours pour $\mathrm{I}_{1} a$ contre $\mathrm{I}, 27$ points et $\mathrm{I}, 49 \mathrm{~kg}$ pour $\mathrm{I}_{2} a$ en Charolais ; 0,65 points et $2,0 \mathrm{I} \mathrm{kg}$ contre 0,49 points et $2,34 \mathrm{~kg}$ respectivement pour les mêmes index en Limousin).

Les deux index $\mathrm{I}_{1} b, \mathrm{I}_{2} b$ soumis à la contrainte de maintien du poids à la naissance conduisent pratiquement aux mêmes résultats dans chacune des races paternelles. Ils sont, comme prévu et surtout en Limousin, moins précis que les index non contraints. Le coût génétique de cette contrainte (pourcentage de diminution par rapport au progrès espéré avec une sélection sans contrainte) sur le poids à deux mois et demi s'avère plus accusé en race limousine qu'en charolaise ( 74 contre $5^{8} \mathrm{p}$. Ioo avec $I_{2} ; 70$ contre 46 p. IOo avec $I_{1}$ ). De plus, l'adjonction de la contrainte entraîne une augmentation de gain sur la valeur bouchère, appréciable surtout en race Limousine. De ce fait, le coût génétique de la contrainte calculé sur la valeur économique globale est plus faible que sur le poids à 75 jours et atteint 22 p. Ioo en Charolais contre $46 \mathrm{p}$. Ioo en Limousin avec l'index $\mathrm{I}_{2}$. Ce coût global peut même être considéré comme surestimé ; il y aurait lieu, de déduire de la valeur du progrès économique global espéré par sélection sur l'index non contraint, le coût imputable à l'accroissement des difficultés de vêlage consécutif à une augmentation du poids à la naissance.

Enfin, il est à remarquer que les index de sélection sans contrainte accordent relativement plus d'importance à la croissance et, en contrepartie, moins à la conformation bouchère en Limousin qu'en Charolais, ainsi qu'en attestent les valeurs du rapport $b_{3} / b_{2}\left(\mathrm{I}, 27\right.$ en Limousin contre 2,36 en Charolais avec $\mathrm{I}_{2} a ; 3,20$ contre $8,7 \mathrm{I}$ avec $I_{1} a$ ). Ces différences de pondération se répercutent par des différences, favorables au Charolais pour le pointage de valeur bouchère et au Limousin pour la croissance, au niveau des gains génétiques, sans toutefois affecter sensiblement le progrès sur la valeur économique globale. Ces différences de comportement entre les deux races sous l'effet d'une sélection par index découlent directement de différences entre elles dans la variabilité génétique de la croissance et de la conformation (tab1. 2). Un coût génétique plus élevé de la contrainte en Limousin qu'en Charolais s'explique par une plus faible héritabilité du poids à 75 jours en Charolais $\left(h^{2}=0,06\right)$ qu'en Limou$\sin \left(h^{2}=0, I 6\right)$, alors que les valeurs de ce coefficient sont pratiquement égales pour le poids à la naissance : $(0,24$ et 0,I9 respectivement en Charolais et Limousin). 
Nos résultats concordent avec ceux de MolinUevo (I97I) établis dans le cadre d'une sélection massale des taureaux utilisés en race pure. Quant à 1'incidence de cette contrainte de maintien du poids à la naissance dans ces deux races, 1'adoption de la contrainte s'accompagne toutefois dans cette situation d'un coût génétique sur le poids à I 80 jours moins élevé (réduction de $5^{8} \mathrm{p}$. Ioo en Limousine et II p. Ioo en Charolais) que celui auquel nous aboutissons sur le poids à 75 jours (respectivement 74 et $5^{8} \mathrm{p}$. Ioo avec $1^{\prime}$ index $\mathrm{I}_{2}$ ). De plus, contrairement au cas du croisement envisagé ici, ces résultats sont liés principalement à une différence des valeurs de l'héritabilité du poids à la naissance — plus élevée pour les veaux mâles Limousins $\left(h^{2}=0,47\right)$ que pour les Charolais $\left(h^{2}=0,22\right)-$, celles du poids au sevrage étant comparables.

\section{TABLEAU 2}

Coefficients de corrélation génétique et d' héritabilité d'après PoujARDIEU et VISSAC, 1968 (sexes réunis)

\begin{tabular}{|c|c|c|c|c|c|c|}
\hline & $\begin{array}{c}\text { Poids } \\
\text { à la naissance }\end{array}$ & $\begin{array}{l}\text { Poids } \\
\text { à } 60 \text { jours }\end{array}$ & $\begin{array}{l}\text { Poids } \\
\text { à } 75 \text { jours }\end{array}$ & $\begin{array}{l}\text { Gain } \\
\text { à } 75 \text { jours }\end{array}$ & Pointage & Prix de vente \\
\hline Prix de vente & 0,02 & 0,19 & 0,14 & 0,08 & 0,78 & 0,14 \\
\hline Pointage & 0,14 & 0,36 & 0,46 & 0,47 & 0,32 & 0,79 \\
\hline $\begin{array}{c}\text { Gain } \\
\text { à } 75 \text { jours }\end{array}$ & 0,14 & 0,8 ' & 0,71 & 0,03 & 0,40 & 0,18 \\
\hline $\begin{array}{l}\text { Poids } \\
\text { à } 75 \text { jours }\end{array}$ & 0,79 & 1,01 & 0,06 & 0,92 & 0,34 & 0,17 \\
\hline $\begin{array}{l}\text { Poids } \\
\text { à } 60 \text { jours }\end{array}$ & 0,75 & 0,09 & 0,98 & 0,87 & 0,34 & 0,17 \\
\hline $\begin{array}{l}\text { Poids } \\
\text { à la naissance }\end{array}$ & 0,24 & 0,74 & 0,68 & 0,36 & 0,06 & 0,01 \\
\hline
\end{tabular}

Les coefficients d'héritabilité sont indiqués dans les cases de la diagonale.

Les valeurs au-dessus de la diagonale concernent la race Charolaise, celles au-dessous la Limousine.

L'analyse de variance a été faite sur 108 taureaux en Charolais et 97 en Limousin.

\section{CONCLUSION}

Cette étude peut apporter certains éléments de réponse au problème du choix d'une indexation rationnelle des mâles des races Charolaise et Limousine destinés au croisement de première génération pour la production de veaux de boucherie.

Elle confirme le bien-fondé de la substitution de la note de pointage au prix 
unitaire de vente dans l'établissement des index de sélection synthétiques qui peuvent être calculés, de façon quasi équivalente quant à leur efficacité théorique, par les produits des valeurs génotypiques du poids à 75 jours d'une part, par l'estimée du prix unitaire à partir du pointage, ou directement par le pointage d'autre part.

Compte tenu des différences des difficultés de vêlage suivant les races paternelles (effet direct du taureau), conditions plus défavorables avec des pères Charolais qu'avec des Limousins, et suivant 1'âge des femelles accouplées aux taureaux de ces races, il semble judicieux d'utiliser l'index sans contrainte plutôt dans la sélection des taureaux Limousins, et dans le cas d'accouplements à des femelles adultes. L'application de la contrainte pourrait, par contre, s'avérer très utile dans le classement des taureaux Charolais destinés aux génisses afin d'infléchir la courbe de croissance de leurs veaux dans un sens favorable. On s'orienterait ainsi vers un classement différentiel des taureaux établi en fonction de leur utilisation suivant l'âge des femelles auxquelles ils sont accouplés. On en viendrait finalement à envisager une évolution différente des races Charolaise et Limousine sous les effets de la sélection, en égard à l'emploi de coefficients de pondération de la musculature et de la croissance spécifiques à chaque race et même de systèmes d'indexation différents (sans contrainte sur l'évolution du poids à la naissance en Limousin, avec contrainte en Charolais).

Il importe néanmoins, dans la perspective d'une application éventuelle de ces recherches, de formuler les critiques qui limitent la portée de nos conclusions. L'hypothèse de liaison directe entre recette et rentabilité est de moins en moins vraie à mesure que se développent les aliments d'allaitement et que les veaux sont abattus de plus en plus lourds ou remplacés par des jeunes bovins, ce qui implique de prendre en considération le coût de l'alimentation. Les deux alternatives envisagées pour le poids à la naissance (sans contrainte et avec progrès nul) constituent, par ailleurs, des solutions extrêmes ; il conviendrait en pratique de fixer une valeur moyenne limite au poids moyen à la naissance, valeur qui varierait avec la race et 1'âge des femelles soumises au croisement. On pourrait déterminer cette valeur pour chaque échantillon de femelles connaissant son ouverture pelvienne pour un risque de difficultés de vêlage fixé a priori. La préđiction des objectifs de sélection qui sont ici des fonctions quadratiques, suscite des difficultés qui sont loin d'être résolues par le recours aux index linéaires classiques établis sous 1'hypothèse de normalité des distributions. Il serait également nécessaire de reprendre les calculs de ces index à partir d'estimations des paramètres génétiques plus précises que celles utilisées ici. Des études théoriques mériteraient donc d'être poursuivies sur ces trois points en les complétant par les vérifications expérimentales nécessaires, notamment en ce qui concerne l'efficacité réelle des méthodes de sélection avec contrainte.

Rę̧u pour publication en octobre 1971.

\section{REMERCIEMENTS}

Nous remercions M. G. LEForT et B. VISSAC pour la lecture critique de notre manuscrit. 


\section{SUMMARY}

\section{METHODS OF DETERMINING SELECTION INDEXES FOR BEEF-BREED BULLS ON THE ECONOMIC VALUE OF THEIR PROGENY}

The selection of beef bulls for commercial crossing has, till now, aimed at maximization of the gross income (weight at 75 days $\times$ sale price of kg liveweight) furnished by the calf at a given age.

Methods of estimating the selection index when the total economic merit is the product of two traits is first discussed. The expected genetic improvement cannot be calculated simply in terms of the percentage of animals selected and the standard deviation of the quadratic index. A simplified process has been adopted in which the linear terms of selection criteria are estimated by the indexes, the quadratic terms being omitted.

Two methods of indexing Charolais and Limousin bulls are then compared using this process (the sale price is estimated by subjective scoring), taking into account the genetic parameters obtained for each breed, in crossing. Changes introduced into the estimation equation by adding the restriction of no genetic change in birth weight have also been studied. Numerical application in the Charolais and Limousin breeds has put the accent on the ponderation differences which may be attributed to each one of the two selected traits in the two breeds. The indexes give relatively less importance to growth relatively to conformation in the Charolais than in the Limousin.

The genetic costs of the restriction on birth-weight change appear to be greater in Limousin than in Charolais. The expected improvement on the 75 day-weight is reduced by about 50 p. 100 for the Charolais sires and by $70 \mathrm{p}$. roo for the Limousin and there is a decrease on the total economic improvement of respectively, 20 and 45 p. Ioo.

\section{RÉFÉRENCES BIBLIOGRAPHIQUES}

Belic M., Ménissier F., I968. Étude de quelques facteurs influençant les difficultés de vêlage en croisement industriel. Annls. Zootech., 17, 107-142.

Cunningham E P., MOen RA., GJedrem T., I97o. Restriction of selection indexes. Biometrics, 26, 67-74.

Elston R. C., r963. A weight free index for the purpose of ranking or selection with respect to several traits at time. Biometrics, 19, 85-97.

Frebling J., Gaillard J., I970. Efficacité du schéma de sélection des mâles de race à viande française en vue de leur utilisation en croisernent industriel. Bull. tech. Dép. Génét. anim. (Inst. natn. Rech. agron., Fr.), no 8 .

HARRIS D. L., I97o. Breeding for efficiency in livestok production : defining the economic objectives. J. Anim. Sci., 30, 860-865.

HAzel L. N., 1943. The genetic basis for constructing selection indexes. Genetics, 28, 476-490.

Kempthorne O., 1957. An introduction to genetic statistics. Wiley, New York, 5 ro pp

KeMpthorne O., NoRdskog A.W., I959. Restricted selection indices. Biometrics, 15, ro-19.

Lehmann R.P., Gaines J. A., Carter R.C., Bovard K. P., Kincaid C. M., I96I. Selection indexes for weanling traits in beef cattle. J. Anim. Sci., 20, 53-57.

Lindholm B., STONAKER H. H., I957 Economic importance of traits and selection indexes for beef cattle. J. Anim. Sci., 14, 979 .

MoAv R., I966. Specialised sire and dam lines. I. Economic evaluation of crossbreds. Anim. Prod., 8, I93-202.

Molinuevo H. A., I970. Variabilité génétique de la croissance avant sevrage dans les races Charolaise et Limousine. Thèse $3^{\text {e }}$ cycle. Génét. quant. appl., Fac. Sci. Paris.

Poujardieu B., Vissac B., I968. Étude biométrique de la croissance et de la valeur bouchère de veaux croisés Charolais et Limousins. I. Paramètres génétiques et phénotypiques. Annls. Zootech., 17, I43-I58.

Rouvier R., I969a. Contribution à l'étude des index sur plusieurs caractères. Thèse $3^{\mathbf{e}}$ cycle. Génét. quant. appl., Fac. Sci. Paris.

Rouvier R., I969 $b$. Pondération des valeurs génotypiques dans la sélection par index sur plusieurs caractères. Biometrics, 25, 295-307. 
Sмrтн C., 1967. A note on the improvement of a trait by selecting on its components. Anim. Prod., 9, I27-I 30 .

Sмiтн H. F., I936. A discriminant function for plant selection. Ann. Eugen., 7, 240-250.

Vissac B., 1964. Méthodes de détermination de l'index génotypique des taureaux d'insémination sur la valeur de leurs veaux de boucherie. Annls. Zootech., 13, 267-275.

Williams J. S., r962. Some statistical properties of a genetic selection index. Biometrika, 49, 325-337. Wilson L. L., Dinkel C. A., Ray D. E., Minyard J. A., r963. Beef cattle selecting indexes involving conformation and weight. J. Anim. Sci., 22, 1086-1o9o.

Wilton J. W., Van Vleck L. D., 1967. Selection for two dependently priced traits. J. A nim. Sci., 26, 890 .

Wilton J. W., Evans D. A., VAN VLECK I. D., I968. Selection indices for quadratic models of total merit. Biometrics, 24, 937-949.

Wilton J. W., VAn Vleck L. D., I968. Selection of dairy cows for economic merit. J. Dairy Sci., 51, I680-I686.

Wilton J. W., Van Vleck L. D., 1969. Sire evaluation for economic merit. J. Dairy Sci., 52, $235-239$. 